\title{
Easy access to cis-1,3-disubstituted cyclopentane 1,4-diphosphines
}

\author{
Pelayo Camps*, Gisela Colet, and Santiago Vázquez \\ Laboratori de Química Farmacèutica (Unitat Associada al CSIC), Facultat de Farmàcia, \\ Universitat de Barcelona, Av. Diagonal 643, E-08028, Barcelona, Spain \\ E-mail: camps@farmacia.far.ub.es
}

\section{Dedicated to Prof. Dr. Roberto A. Rossi on the occasion of his $60^{\text {th }}$ anniversary}

(received 19 Dec 02; accepted 07 Mar 03; published on the web 24 Mar 03)

\begin{abstract}
cis-1-(Diphenylphosphino)-3-(diphenylphosphinomethyl)cyclopentane

(13a) and the corresponding dicyclopentylphosphino derivative 13b have been readily obtained in high yield from 2-cyclopentenone by a five-step sequence which takes advantage of the electrophilic character of positions 1 and 3 of 2-cyclopentenone to introduce the substituents, while their relative cis-configuration is established by diastereoselective hydrogenation of the mixture of isomeric alkenes 12a or $\mathbf{1 2 b}$.
\end{abstract}

Keywords: Addition reactions, carbocycles, diastereoselectivity, hydrogenation, phosphorus

\section{Introduction}

As part of our current interest on the synthesis of carbocyclic analogs of cis-MCCPM (Figure $1),{ }^{1}$ we have recently described the synthesis of bisphosphinoyl compounds 5a-c in racemic form (Scheme 1). ${ }^{2}$ Also, compound (6a) was prepared by dehydroxylation of $\mathbf{5 a}$ by using the Barton procedure. However, the synthesis of these compounds has serious drawbacks: (a) multi-step reaction sequence; (b) low overall yield; and (c) stereoselectivity problems in the crucial step (nucleophilic opening of epoxide 4 with the lithium derivative of methyldiphenylphosphine oxide, and (d) column chromatography of the acetates derived from the mixture of $\mathbf{5}$ and $\mathbf{7}$ was required to isolate the desired minor cis-stereoisomers.

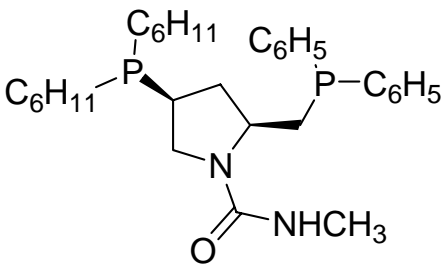


Figure 1. $(S, S)$-cis-MCCPM.

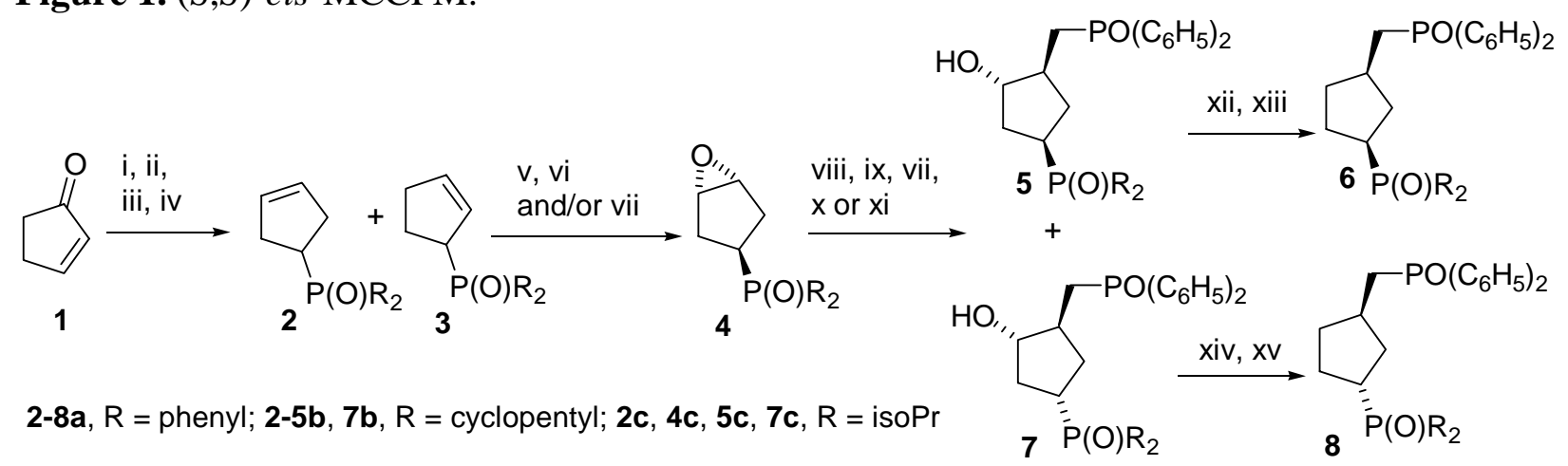

Scheme 1. Reagents and conditions: (i) $\mathrm{ClPR}_{2}, \mathrm{AcOH}, 4 \AA$ molecular sieves, r.t., 2 h; (ii) $\mathrm{NaBH}_{4}, \mathrm{MeOH}$; (iii) MsCl, Et 3 N, DMAP (10\%), $\mathrm{CH}_{2} \mathrm{Cl}_{2}$; (iv) Pyrolysis; (v) m-Chloroperbenzoic acid, $\mathrm{CH}_{2} \mathrm{Cl}_{2}$; (vi) $\mathrm{KOH}$, EtOH; (vii) Silica gel column chromatography; (viii) Lithium derivative of methyldiphenylphosphine oxide (9) [from 9 and 1.6 M n-BuLi (in hexanes)], THF, r.t. (3 h) and reflux (15 h); (ix) $\mathrm{Ac}_{2} \mathrm{O}$, reflux, 2 h; (x) NaMeO, MeOH, reflux, 2 h; (xi) KCN, EtOH, reflux, 12 h; (xii) Thiocarbonyldiimidazole, toluene, reflux, 1 h; (xiii) $n$-Bus $\mathrm{SnH}$, AIBN, toluene, reflux, 1 h. (xiv) Thiocarbonyldiimidazole, $\mathrm{CH}_{2} \mathrm{Cl}_{2}$, reflux, $4 \mathrm{~h}$; (xv) $n-\mathrm{Bu}_{3} \mathrm{SnH}$, AIBN, benzene, reflux, $6 \mathrm{~h}$.

\section{Results and Discussion}

Herein we describe a straightforward diastereoselective synthesis of $\mathbf{6 a}$ and the corresponding 3(dicyclopentylphosphinoyl) derivative (6b), starting from 2-cyclopentenone (1) (Scheme 2) and their conversion into the corresponding diphosphines, 13a and 13b, respectively. Since positions 1 and 3 of 2-cyclopentenone are electrophilic, both substituents of the desired diphosphines (13) could be introduced by appropriate nucleophilic addition reactions. It is known that chlorodiphenylphosphine reacts with acyclic $\alpha, \beta$-unsaturated ketones in anhydrous acetic acid to give a $\beta$-(diphenylphosphinoyl)ketone, ${ }^{3}$ while we have recently described ${ }^{2}$ the synthesis of 10a and 10b by using the same kind of reaction. Also, configurationally stable lithiated P-chiral disubstituted phosphine oxides have been added (Michael reaction) with high diastereoselectivity to 2-cyclopentenone. ${ }^{4}$ Moreover, examples of nucleophilic additions of the lithium derivative of methyldiphenylphosphine oxide (9) and related derivatives to ketones are also known. ${ }^{5}$

Initial attempts to carry out the nucleophilic addition of the lithium derivative of 9 to cyclopentanone (10a) failed, probably due to the water present in compound (10a). When 10a was made anhydrous by azeotropic distillation of water with toluene and then it was reacted with the lithium derivative of $\mathbf{9}$, the corresponding addition product (11a) was obtained in high yield. 
Only one stereoisomer, probably the one derived from the attack of the nucleophile on the less hindered carbonyl face, was observed.

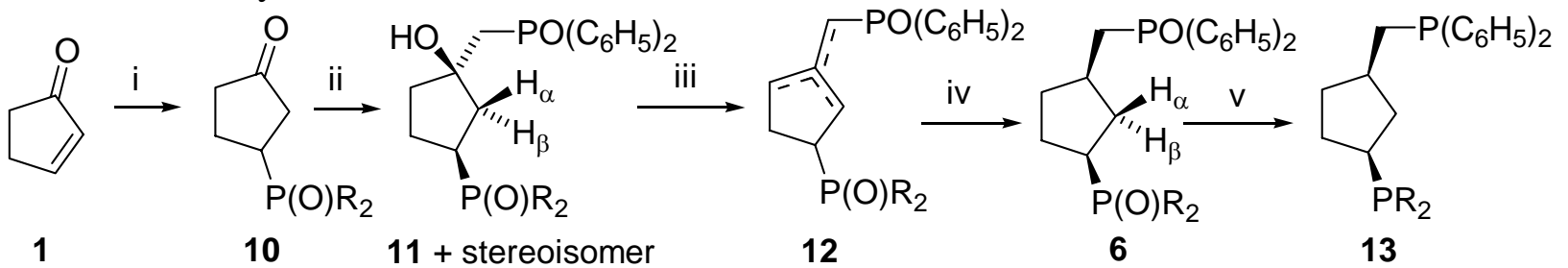

6a, 10-13a, $\mathrm{R}=\mathrm{C}_{6} \mathrm{H}_{5} ; \mathbf{6 b}, \mathbf{1 0 - 1 3 b}, \mathrm{R}=$ cyclopentyl

Scheme 2. Reagents and conditions: (i) see reference 2; (ii) Lithium derivative of methyldiphenylphosphine oxide (9) [from 9 and 1.6 M n-BuLi (in hexanes)], THF, r.t. (3 h) and reflux (15 h); (iii) Concentrated $\mathrm{H}_{2} \mathrm{SO}_{4}$, THF, reflux, 3-6 d; (iv) $\mathrm{H}_{2}$, 5\% Pd-C, MeOH, 1 atm, 3-6 d; (v) $\mathrm{HSiCl}_{3}, \mathrm{Et}_{3} \mathrm{~N}, \mathrm{CH}_{3} \mathrm{CN}$, reflux, $3 \mathrm{~h}$.

Once the diphenylphosphinoyl and diphenylphosphinoylmethyl substituents were introduced, the hydroxyl group of compound 11a was removed in order to establish the relative cisconfiguration of the substituents. To this end, compound 11a was dehydrated, which required quite drastic conditions ( 2 mol of concentrated $\mathrm{H}_{2} \mathrm{SO}_{4}$ per mol of 11a, in THF under reflux for 3 days). The product thus obtained (93\% yield) consisted of a mixture of regio- and stereo-isomers (12a) which was submitted without separation to hydrogenation under standard conditions. Fortunately, from the hydrogenation, only compound 6a was obtained in $83 \%$ yield. The relative cis-configuration of this compound was assigned by comparison of its ${ }^{1} \mathrm{H}$ and ${ }^{13} \mathrm{C}$ NMR data with those of a reference sample of $\mathbf{6 a}$, prepared by the synthetic sequence of scheme 1, whose relative cis-configuration had unequivocally been established by X-ray diffraction analysis. ${ }^{2}$ Moreover, the ${ }^{1} \mathrm{H}$ and ${ }^{13} \mathrm{C}$ NMR data of $6 \mathbf{a}$ differ from those of the corresponding transstereoisomer (8a, scheme 1). ${ }^{2}$

Similarly, reaction of $\mathbf{1 0 b}^{2}$ with the lithium derivative of $\mathbf{9}$ gave in good yield a mixture of 11b and its stereoisomer in an approximate ratio of 5:1, respectively. The main component was assumed to be $\mathbf{1 1 b}$. Dehydration of the mixture of $\mathbf{1 1 b}$ and its stereoisomer gave a regio- and stereo-isomeric mixture of alkenes $\mathbf{1 2 b}$, which on hydrogenation gave $\mathbf{6 b}$, as a highly hygroscopic solid, whose melting point could not be determined. The relative cis-configuration of $\mathbf{6 b}$ was established by comparison of its ${ }^{1} \mathrm{H}$ and ${ }^{13} \mathrm{C}$ NMR spectra with those of $6 \mathbf{a}$. Compounds $\mathbf{6 a}$ and $\mathbf{6 b}$ were reduced in high yield to the corresponding diphosphines 13a and 13b by reaction with trichlorosilane.

The new compounds 11a, 11b + stereoisomer, and $\mathbf{6 b}$ have been fully characterized by spectroscopic means (IR, ${ }^{1} \mathrm{H},{ }^{13} \mathrm{C}$ and ${ }^{31} \mathrm{P} \mathrm{NMR}, \mathrm{MS}$ ) and elemental analysis, while diphosphines 13a and 13b have been characterized by NMR spectroscopy $\left({ }^{1} \mathrm{H},{ }^{13} \mathrm{C}\right.$ and $\left.{ }^{31} \mathrm{P}\right)$. In general, assignment of the NMR spectra has been carried out with the aid of COSY ${ }^{1} \mathrm{H} /{ }^{1} \mathrm{H}$, HETCOR ${ }^{1} \mathrm{H} /{ }^{13} \mathrm{C}$ and NOESY experiments. 
In conclusion, the unexpected stereoselective hydrogenation of the mixture of alkenes 12a and 12b to the cis-derivatives $\mathbf{6 a}$ and $\mathbf{6 b}$, opens the way to the synthesis of a new family of cis-1,3disubstituted cyclopentane 1,4-diphosphines, which might be of interest to prepare new chiral catalysts. Work is in progress to prepare and isolate Rh (I) complexes derived from diphosphines 13a and 13b, to study their catalytic activity in hydrogenation reactions.

\section{Experimental Section}

General Procedures. Melting points were determined with a MFB 595010 M Gallenkamp melting point apparatus. $500 \mathrm{MHz}{ }^{1} \mathrm{H}$ NMR spectra were recorded on a Varian VXR 500 spectrometer, $75.4 \mathrm{MHz}{ }^{13} \mathrm{C}$ NMR spectra were taken on a Varian Gemini 300 and $121.4 \mathrm{MHz}$ ${ }^{31} \mathrm{P}$ NMR on a Varian Unity 300 Plus, always in $\mathrm{CDCl}_{3}$ solution. ${ }^{1} \mathrm{H}$ and ${ }^{13} \mathrm{C} \mathrm{NMR}$ chemical shifts $(\delta)$ are reported in ppm with respect to internal tetramethylsilane (TMS) and ${ }^{31} \mathrm{P}$ NMR chemical shifts $(\delta)$ are reported in ppm relative to $85 \% \mathrm{H}_{3} \mathrm{PO}_{4}$ as external standard. The multiplicity of the signals is: s, singulet; d, doublet; t, triplet; m, multiplet. For the different compounds, the terms $\mathrm{H}_{\alpha}$ or $\mathrm{H}_{\beta}$ are assigned to hydrogen atoms which are cis or trans relative to the reference substituent (usually at position 1), respectively. IR spectra were recorded on a FT/IR Perkin-Elmer spectrometer, model 1600; only significant absorption bands are given. Routine MS spectra were taken on a Hewlett-Packard 5988A spectrometer, the sample was introduced directly or through a gas chromatograph, Hewlett-Packard model 5890 Series II, equipped with a 30-meter HP-5 (5\% diphenyl-95\% dimethyl-polysiloxane) column and the electron impact technique $(70 \mathrm{eV})$. Only significant ions are given: those with higher relative abundance, except for the ions with higher $\mathrm{m} / \mathrm{z}$ values. NMR and routine MS spectra were performed at the Serveis Científico-Tècnics of the University of Barcelona, while elemental analyses were carried out at the Microanalysis Service of the IIQAB (CSIC, Barcelona, Spain).

c-3-(Diphenylphosphinoyl)-1-[(diphenylphosphinoyl)methyl]-r-1-cyclopentanol (11a). To a cold (ice-bath) solution of methyldiphenylphosphine oxide (98\%, $580 \mathrm{mg}, 2.63 \mathrm{mmol}$ ) in anhydrous THF $(15 \mathrm{~mL})$ was added dropwise $n$-butyllithium $(2.36 \mathrm{~mL}, 1.6 \mathrm{M}$ in hexanes, 3.78 $\mathrm{mmol}$ ) and the suspension was stirred at $0^{\circ} \mathrm{C}$ for $45 \mathrm{~min}$. The suspension was cooled to $-78^{\circ} \mathrm{C}$ and a solution of anhydrous ketone 10a $(747 \mathrm{mg}, 2.63 \mathrm{mmol}$, azeotropic distillation of the water content with toluene in a Dean-Stark equipment) in THF (25 mL) was added dropwise. After $3 \mathrm{~h}$ at room temperature, the mixture was heated under reflux for $15 \mathrm{~h}$. The mixture was allowed to cool to room temperature, saturated aqueous solution of $\mathrm{NH}_{4} \mathrm{Cl}(19 \mathrm{~mL})$ was added, and the organic phase was separated and evaporated to dryness in vacuo. The residue was taken in $\mathrm{H}_{2} \mathrm{O}$ (35 mL) and the solution was extracted with $\mathrm{CH}_{2} \mathrm{Cl}_{2}(3 \times 37 \mathrm{~mL})$. The combined organic phases were dried $\left(\mathrm{Na}_{2} \mathrm{SO}_{4}\right)$, filtered and concentrated in vacuo to give 11a (950 mg, $72 \%$ yield) as an orange-brown viscous oil. The analytical sample of 11a was obtained as a colorless solid by crystallization (ethyl acetate), m.p. $179-180^{\circ} \mathrm{C}$. IR (KBr) 3425 (OH st), 1197, 1158, 1119 (P=O 
st) $\mathrm{cm}^{-1}$; ${ }^{1} \mathrm{H}$ NMR 7.81-7.63 (complex signal, 8H, Ar-Hortho), 7.49-7.36 (complex signal, 12H, Ar- $\mathrm{H}_{\text {meta }}$, Ar- $\mathrm{H}_{\text {para }}$ ), 5.80-5.20 (broad signal, 1H, OH), 3.01-2.92 (m, 1H, 3-H), 2.82 (dd, 1H, $J=15.0 \mathrm{~Hz}, J=12.0 \mathrm{~Hz}$ ) and $2.76\left(\mathrm{dd}, 1 \mathrm{H}, J=15.0 \mathrm{~Hz}, J^{\prime}=11.0 \mathrm{~Hz}\right)\left(\mathrm{CH}_{2}-\mathrm{P}\right), 2.52$ (ddd, 1H, $J=19.0 \mathrm{~Hz}, J^{\prime}=14.5 \mathrm{~Hz}, J^{\prime \prime}=12.0 \mathrm{~Hz}, 2-\mathrm{H}_{\beta}$ ), 2.10-1.95 (complex signal, 2H, 2- $\mathrm{H}_{\alpha}, 4-\mathrm{H}_{\alpha}$ ), 1.82-1.70 (complex signal, 3H, 4- $\left.\mathrm{H}_{\beta}, 5-\mathrm{H}_{\alpha}, 5-\mathrm{H}_{\beta}\right)$; ${ }^{13} \mathrm{C}$ NMR 134.4 (C, d, ${ }^{1} J_{\mathrm{CP}}=98.2 \mathrm{~Hz}$ ), 134.0 $\left(\mathrm{C}, \mathrm{d},{ }^{1} J_{\mathrm{CP}}=98.2 \mathrm{~Hz}\right), 132.1\left(\mathrm{C}, \mathrm{d},{ }^{1} J_{\mathrm{CP}}=98.8 \mathrm{~Hz}\right), 131.6\left(\mathrm{C}, \mathrm{d},{ }^{1} J_{\mathrm{CP}}=98.3 \mathrm{~Hz}\right)\left(\mathrm{Ar}-\mathrm{C}_{i p s o}\right), 131.7$ (2 $\left.\mathrm{CH}, \mathrm{d},{ }^{4} J_{\mathrm{CP}}=2.2 \mathrm{~Hz}\right), 131.4\left(\mathrm{CH}, \mathrm{d},{ }^{4} J_{\mathrm{CP}}=2.2 \mathrm{~Hz}\right)$ and $131.3\left(\mathrm{CH}, \mathrm{d},{ }^{4} J_{\mathrm{CP}}=2.5 \mathrm{~Hz}\right)\left(\mathrm{Ar}-\mathrm{CH}_{\text {para }}\right)$, $130.8\left(\mathrm{CH}, \mathrm{d},{ }^{2} J_{\mathrm{CP}}=9.1 \mathrm{~Hz}\right), 130.6\left(\mathrm{CH}, \mathrm{d},{ }^{2} J_{\mathrm{CP}}=9.6 \mathrm{~Hz}\right)$ and $130.5\left(\mathrm{CH}, \mathrm{d},{ }^{2} J_{\mathrm{CP}}=10.2 \mathrm{~Hz}\right)(\mathrm{Ar}-$ $\mathrm{CH}_{\text {ortho }}$ ), $128.6\left(2 \mathrm{CH}, \mathrm{d},{ }^{3} J_{\mathrm{CP}}=11.1 \mathrm{~Hz}\right), 128.4\left(\mathrm{CH}, \mathrm{d},{ }^{3} J_{\mathrm{CP}}=11.2 \mathrm{~Hz}\right)$ and $128.3(\mathrm{CH}, \mathrm{d}$, ${ }^{3} J_{\mathrm{CP}}=11.7 \mathrm{~Hz}$, $\left(\mathrm{Ar}-\mathrm{CH}_{\text {meta }}\right), 78.9\left(\mathrm{C}, \mathrm{t},{ }^{2} J_{\mathrm{CP}}={ }^{3} J_{\mathrm{CP}}=2.0 \mathrm{~Hz}, \mathrm{C} 1\right), 41.6\left(\mathrm{CH}_{2}, \mathrm{t},{ }^{2} J_{\mathrm{CP}}={ }^{3} J_{\mathrm{CP}}=5.6 \mathrm{~Hz}\right.$, C5), $39.8\left(\mathrm{CH}_{2}\right.$, broad s, C2), $38.6\left(\mathrm{CH}_{2}, \mathrm{~d},{ }^{1} J_{\mathrm{CP}}=70.3 \mathrm{~Hz}, \mathrm{CH}_{2}-\mathrm{P}\right), 34.3\left(\mathrm{CH}, \mathrm{d},{ }^{1} J_{\mathrm{CP}}=72.5 \mathrm{~Hz}\right.$, C3), $23.7\left(\mathrm{CH}_{2}, \mathrm{~s}, \mathrm{C} 4\right) ;{ }^{31} \mathrm{P}$ NMR $32.3\left[\mathrm{PO}\left(\mathrm{C}_{6} \mathrm{H}_{5}\right)_{2}\right], 22.0\left[\mathrm{CH}_{2} \mathrm{PO}\left(\mathrm{C}_{6} \mathrm{H}_{5}\right)_{2}\right]$; MS (EI), m/z (\%): $500 \quad\left(\mathrm{M}^{++}, \quad 3\right), 299 \quad\left[\left[\mathrm{M}-\mathrm{PO}\left(\mathrm{C}_{6} \mathrm{H}_{5}\right)_{2}\right]^{+}, \quad 76\right], \quad 281 \quad\left[\left[\mathrm{M}-\mathrm{H}_{2} \mathrm{O}-\mathrm{PO}\left(\mathrm{C}_{6} \mathrm{H}_{5}\right)_{2}\right]^{+}, \quad 20\right], \quad 215$ $\left[\left[\mathrm{CH}_{2} \mathrm{PO}\left(\mathrm{C}_{6} \mathrm{H}_{5}\right)_{2}\right]^{+}, 34\right], 202$ (46), 201 [[PO( $\left.\left(\mathrm{C}_{6} \mathrm{H}_{5}\right)_{2}\right]^{+}$, 100]. Anal. Calcd. for $\mathrm{C}_{30} \mathrm{H}_{30} \mathrm{O}_{3} \mathrm{P}_{2}$ : C, 71.99; H: 6.05. Found: C, 72.04; H, 6.11.

Mixture of $\boldsymbol{c}$-3-(dicyclopentylphosphinoyl)-1-[(diphenylphosphinoyl)methyl]-r-1- cyclopentanol (11b) and $t$-3-stereoisomer. From methyldiphenylphosphine oxide (98\%, $4.19 \mathrm{~g}, 19.0 \mathrm{mmol})$ in anhydrous THF (100 mL), $n$-butyllithium (1.6M in hexanes, $19.0 \mathrm{~mL}, 30.4 \mathrm{mmol})$ and a solution of anhydrous ketone 10b (5.0 g, $18.6 \mathrm{mmol})$ in THF $(100 \mathrm{~mL})$ and following the procedure described for 10a, a mixture of $\mathbf{1 1 b}$ and its stereoisomer in the approximate ratio $\mathbf{1 1 b}$ stereoisomer of 5:1, was obtained (6.57 g, 73\% yield) as a brown foamy solid. The analytical sample of this mixture was obtained as a colorless solid by crystallization (ethyl acetate), m.p. 163-164º C. IR (KBr) 3420 (OH st), 1157, 1119 (P=O st) cm ${ }^{-1}$. MS (EI), m/z (\%): 484 ( $\left.\mathrm{M}^{+}, 2\right)$, $466\left[\left(\mathrm{M}-\mathrm{H}_{2} \mathrm{O}\right)^{++}, 1\right], 416\left[\left(\mathrm{M}-\mathrm{C}_{5} \mathrm{H}_{8}\right)^{++}, 9\right], 347\left[\left(\mathrm{M}-\mathrm{C}_{5} \mathrm{H}_{8}-\mathrm{C}_{5} \mathrm{H}_{9}\right)^{+}, 16\right], 299\left\{\left[\mathrm{M}-\mathrm{PO}\left(\mathrm{C}_{5} \mathrm{H}_{9}\right)_{2}\right]^{+}\right.$, 98\}, $283\left\{\left[\mathrm{M}-\mathrm{PO}\left(\mathrm{C}_{6} \mathrm{H}_{5}\right)_{2}\right]^{+}, 14\right\}, 281\left\{\left[\mathrm{M}-\mathrm{PO}\left(\mathrm{C}_{5} \mathrm{H}_{9}\right)_{2}-\mathrm{H}_{2} \mathrm{O}\right]^{+}, 42\right\}, 215\left\{\left[\mathrm{CH}_{2} \mathrm{PO}\left(\mathrm{C}_{6} \mathrm{H}_{5}\right)_{2}\right]^{+}, 77\right\}$, 201 [PO $\left(\mathrm{C}_{6} \mathrm{H}_{5}\right)_{2}$, 100]. Anal. Calcd. for $\mathrm{C}_{28} \mathrm{H}_{38} \mathrm{O}_{3} \mathrm{P}_{2}$ : C, 69.40; H, 7.91. Found: C, 69.38; H, 7.95. Data of 11b from the spectra of the mixture: ${ }^{1} \mathrm{H}$ NMR 7.78-7.72 (complex signal, $4 \mathrm{H}, \mathrm{Ar}-\mathrm{H}_{\text {ortho), }}$ ) 7.51-7.41 (complex signal, 6H, Ar- $\mathrm{H}_{\text {meta }}, \mathrm{Ar}-\mathrm{H}_{\text {para }}$ ), 5.31 (broad s, 1H, OH), 2.77 (dd, J=15.0 $\mathrm{Hz}, J=10.0 \mathrm{~Hz}, 1 \mathrm{H})$ and $2.72\left(\mathrm{dd}, J=15.0 \mathrm{~Hz}, J^{\prime}=10.0 \mathrm{~Hz}, 1 \mathrm{H}\right)\left(\mathrm{CH}_{2}-\mathrm{P}\right), 2.30-2.21(\mathrm{~m}, 1 \mathrm{H}, 3-\mathrm{H})$, 2.20-1.90 (complex signal, 5H, 2 cyclopentyl $\mathrm{CH}, 2-\mathrm{H}_{\alpha}, 2-\mathrm{H}_{\beta}, 4-\mathrm{H}_{\alpha}$ ), 1.89-1.50 (complex signal, 19H, cyclopentyl $\left.\mathrm{CH}_{2}, 4-\mathrm{H}_{\beta}, 5-\mathrm{H}_{\alpha}, 5-\mathrm{H}_{\beta}\right) ;{ }^{13} \mathrm{C}$ NMR 133.7 (C, d, ${ }^{1} J_{\mathrm{CP}}=99.2 \mathrm{~Hz}, \mathrm{Ar}-\mathrm{C}_{i p s o}$ ), $131.6\left(\mathrm{CH}\right.$, broad s, Ar- $\left.\mathrm{CH}_{\text {para }}\right), 130.4\left(\mathrm{CH}, \mathrm{d},{ }^{2} J_{\mathrm{CP}}=9.7 \mathrm{~Hz}, \mathrm{Ar}-\mathrm{CH}_{\text {ortho }}\right), 128.52(\mathrm{CH}$, d, $\left.{ }^{3} J_{\mathrm{CP}}=12.2 \mathrm{~Hz}\right)$ and $128.45\left(\mathrm{CH}, \mathrm{d},{ }^{3} J_{\mathrm{CP}}=11.7 \mathrm{~Hz}\right)\left(\mathrm{Ar}-\mathrm{CH}_{\text {meta }}\right), 79.3\left(\mathrm{CH}, \mathrm{t},{ }^{2} J_{\mathrm{CP}}={ }^{3} J_{\mathrm{CP}}=4.8 \mathrm{~Hz}\right.$, C1), $41.6\left(\mathrm{CH}_{2}, \mathrm{t},{ }^{3} J_{\mathrm{CP}}={ }^{3} J_{\mathrm{CP}}=6.9 \mathrm{~Hz}, \mathrm{C} 5\right), 40.6\left(\mathrm{CH}_{2}, \mathrm{~d},{ }^{3} J_{\mathrm{CP}}=5.0 \mathrm{~Hz}, \mathrm{C} 2\right), 38.7\left(\mathrm{CH}_{2}, \mathrm{~d}\right.$, $\left.{ }^{1} J_{\mathrm{CP}}=69.9 \mathrm{~Hz}, \mathrm{CH}_{2}-\mathrm{P}\right), 36.4\left(\mathrm{CH}, \mathrm{d},{ }^{1} J_{\mathrm{CP}}=66.3 \mathrm{~Hz}\right)$ and $36.2\left(\mathrm{CH}, \mathrm{d},{ }^{1} J_{\mathrm{CP}}=66.3 \mathrm{~Hz}\right)$ (cyclopentyl $\mathrm{CH}), 34.8\left(\mathrm{CH}, \mathrm{d},{ }^{1} J_{\mathrm{CP}}=64.3 \mathrm{~Hz}, \mathrm{C} 3\right), 27.1-27.0\left(\mathrm{CH}_{2}\right.$, cyclopentyl $\mathrm{C} 2$ and $\left.\mathrm{C} 5\right), 26.1-25.8\left(\mathrm{CH}_{2}\right.$, cyclopentyl C3 and C4), $24.6\left(\mathrm{CH}_{2}, \mathrm{~d},{ }^{2} J_{\mathrm{CP}}=2.0 \mathrm{~Hz}, \mathrm{C} 4\right) ;{ }^{31} \mathrm{P}$ NMR 54.8 [PO $\left.\left(\mathrm{C}_{5} \mathrm{H}_{9}\right)_{2}\right], 28.5$ $\left[\mathrm{PO}\left(\mathrm{C}_{6} \mathrm{H}_{5}\right)_{2}\right]$. Data of the stereoisomer of $\mathbf{1 1 b}$ from the spectrum of the mixture: ${ }^{31} \mathrm{P}$ NMR 52.0 [PO $\left.\left(\mathrm{C}_{5} \mathrm{H}_{9}\right)_{2}\right], 31.2\left[\mathrm{PO}\left(\mathrm{C}_{6} \mathrm{H}_{5}\right)_{2}\right]$. 
cis-1-(Diphenylphosphinoyl)-3-[(diphenylphosphinoyl)methyl]cyclopentane (6a). a) Dehydration of 11a to the mixture of alkenes 12a. To a solution of 11a (1.46 g, $2.92 \mathrm{mmol})$ in THF (60 mL), concentrated $\mathrm{H}_{2} \mathrm{SO}_{4}(0.32 \mathrm{~mL}, 5.84 \mathrm{mmol})$ was added and the mixture was stirred under reflux for 3 days. The mixture was allowed to cool to room temperature and the solvent was evaporated in vacuo. The residue was dissolved in $\mathrm{CH}_{2} \mathrm{Cl}_{2}(30 \mathrm{~mL})$, washed with $\mathrm{NaHCO}_{3}$ (saturated aqueous solution, $3 \times 20 \mathrm{~mL}$ ), dried $\left(\mathrm{Na}_{2} \mathrm{SO}_{4}\right)$, filtered and concentrated to dryness in vacuo to give the mixture of alkenes 12a as a brown foamy solid (1.31 g, 93\% yield).

b) Hydrogenation of the mixture of alkenes 12a. To a solution of the mixture of alkenes 12a (557 mg, $1.16 \mathrm{mmol}$ ) in methanol (25 mL), Pd-C (5\% Pd, 54\% water content, $223 \mathrm{mg}$ ) was added and the mixture was vigorously stirred under hydrogen $(1 \mathrm{~atm})$ for 3 days. The suspension was filtered and the filtrate was concentrated to dryness in vacuo to give $6 \mathbf{6 a}$ (464 mg, 83\% yield) as a pale yellow foamy solid, whose ${ }^{1} \mathrm{H}$ and ${ }^{13} \mathrm{C}$ NMR spectra coincide with those of a sample of $6 \mathbf{a}$, previously obtained by a different synthetic procedure. ${ }^{2}$

cis-1-(Dicyclopentylphosphinoyl)-3-[(diphenylphosphinoyl)methyl]cyclopentane (6b). a) Dehydration of $\mathbf{1 1 b}$ to the mixture of alkenes 12b. From a mixture of $\mathbf{1 1 b}$ and its stereoisomer (6.57 g, 13.6 mmol) and concentrated $\mathrm{H}_{2} \mathrm{SO}_{4}(1.48 \mathrm{~mL}, 27.2 \mathrm{mmol})$ in THF (150 mL), following the procedure described for 11a, but stirring under reflux for 6 days, a mixture of alkenes $\mathbf{1 2 b}$ was obtained as a brown oil (5.43 g, 86\% yield).

b) Hydrogenation of the mixture of alkenes 12b. To a solution of the mixture of alkenes $\mathbf{1 2 b}$ (1.33 g, $2.85 \mathrm{mmol}$ ) in methanol (65 mL) Pd-C (5\% Pd, 54\% water content, $0.55 \mathrm{~g}$ ) was added and the mixture was vigorously stirred under a hydrogen atmosphere for 6 days. The suspension was filtered and the filtrate was concentrated to dryness in vacuo to give $\mathbf{6 b}$ (1.19 g, 89\% yield) as a pale yellow foamy solid. An analytical sample of $\mathbf{6 b}$ was obtained by crystallization (ethyl acetate), as a very hygroscopic white solid, whose m.p. could not be determined. IR (KBr) 1159,

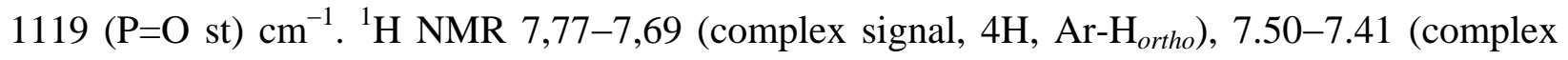
signal, 6H, Ar- $\mathrm{H}_{\text {meta }}$, Ar- $\mathrm{H}_{\text {para }}$ ), 2.48-2.28 [complex signal, 3H, 3-H, $\mathrm{CH}_{2} \mathrm{P}$ ], 2.14-1.93 (complex signal, 4H, cyclopentyl $\mathrm{CH}, 1-\mathrm{H}, 5-\mathrm{H}_{\alpha}$ ), 1.93-1.45 (complex signal, 19H, 8 cyclopentyl $\mathrm{CH}_{2}$, 2$\mathrm{H}_{\beta}$, 4- $\mathrm{H}_{\beta}, 5-\mathrm{H}_{\beta}$ ), 1.45-1.30 (complex signal, $2 \mathrm{H}, 2-\mathrm{H}_{\alpha}, 4-\mathrm{H}_{\alpha}$ ); ${ }^{13} \mathrm{C}$ NMR $133.4\left(\mathrm{C}, \mathrm{d},{ }^{1} J_{\mathrm{CP}}=97.7\right.$ $\mathrm{Hz})$ and $133.2\left(\mathrm{C}, \mathrm{d},{ }^{1} J_{\mathrm{CP}}=97.7 \mathrm{~Hz}\right)\left(\mathrm{Ar}-\mathrm{C}_{i p s o}\right), 131.5\left(\mathrm{CH}, \mathrm{d},{ }^{4} J_{\mathrm{CP}}=3.0 \mathrm{~Hz}\right)$ and $131.4(\mathrm{CH}, \mathrm{d}$, $\left.{ }^{4} J_{\mathrm{CP}}=2.6 \mathrm{~Hz}\right)\left(\mathrm{Ar}-\mathrm{CH}_{\text {para }}\right), 130.6\left(\mathrm{CH}, \mathrm{d},{ }^{2} J_{\mathrm{CP}}=9.1 \mathrm{~Hz}\right)$ and $130.4\left(\mathrm{CH}, \mathrm{d},{ }^{2} J_{\mathrm{CP}}=9.1 \mathrm{~Hz}\right)(\mathrm{Ar}-$ $\left.\mathrm{CH}_{\text {ortho }}\right), 128.5\left(\mathrm{CH}, \mathrm{d},{ }^{3} J_{\mathrm{CP}}=11.2 \mathrm{~Hz}, 2 \mathrm{Ar}-\mathrm{CH}_{\text {meta }}\right), 36.9\left(\mathrm{CH}, \mathrm{d},{ }^{1} J_{\mathrm{CP}}=66.3 \mathrm{~Hz}\right)$ and $36.5(\mathrm{CH}, \mathrm{d}$, ${ }^{1} J_{\mathrm{CP}}=65.3 \mathrm{~Hz}$ ) (cyclopentyl $\left.\mathrm{CH}\right), 35.8\left(\mathrm{CH}, \mathrm{d},{ }^{1} J_{\mathrm{CP}}=64.8 \mathrm{~Hz}, \mathrm{C} 1\right), 35.7\left(\mathrm{CH}_{2}, \mathrm{~d},{ }^{3} J_{\mathrm{CP}}=7.1 \mathrm{~Hz}\right.$, C2), $34.7\left(\mathrm{CH}, \mathrm{dd},{ }^{3} J_{\mathrm{CP}}=11.3 \mathrm{~Hz},{ }^{2} J_{\mathrm{CP}}=3.8 \mathrm{~Hz}, \mathrm{C} 3\right), 34.6\left(\mathrm{CH}_{2}, \mathrm{~d},{ }^{1} J_{\mathrm{CP}}=71.3 \mathrm{~Hz}, \mathrm{CH}_{2} \mathrm{P}\right), 33.6$ $\left(\mathrm{CH}_{2}\right.$, dd, $\left.{ }^{3} J_{\mathrm{CP}}=9.1 \mathrm{~Hz},{ }^{3} J_{\mathrm{CP}}=6.6 \mathrm{~Hz}, \mathrm{C} 4\right), 27.1\left(2 \mathrm{CH}_{2}\right.$, broad s) and $26.9\left(\mathrm{CH}_{2}\right.$, broad $\left.\mathrm{s}\right)$ (cyclopentyl C2 and C5), $26.8\left(\mathrm{CH}_{2}\right.$, broad s), $26.1\left(2 \mathrm{CH}_{2}, \mathrm{~d},{ }^{3} \mathrm{~J}_{\mathrm{CP}}=6.0 \mathrm{~Hz}\right)$ and $25.9\left(2 \mathrm{CH}_{2}, \mathrm{~d}\right.$, ${ }^{3} \mathrm{~J}_{\mathrm{CP}}=6.0 \mathrm{~Hz}$ ) (cyclopentyl C3 and C4), $24.8\left(\mathrm{CH}_{2}\right.$, broad s, C5); ${ }^{31} \mathrm{P}$ NMR $28.3\left[\mathrm{PO}\left(\mathrm{C}_{6} \mathrm{H}_{5}\right)_{2}\right], 55.7$ $\left[\mathrm{PO}\left(\mathrm{C}_{5} \mathrm{H}_{9}\right)_{2}\right] . \mathrm{MS}(\mathrm{EI}), \mathrm{m} / \mathrm{e}(\%): 469\left[(\mathrm{M}+\mathrm{H})^{+}, 2\right], 468\left(\mathrm{M}^{++}, 1\right), 400\left[\left(\mathrm{M}-\mathrm{C}_{5} \mathrm{H}_{8}\right)^{\cdot+}, 13\right], 331$ $\left[\left(\mathrm{M}-\mathrm{C}_{5} \mathrm{H}_{9}-\mathrm{C}_{5} \mathrm{H}_{8}\right)^{+}, \quad 55\right], 283\left\{\left[\mathrm{M}-\mathrm{PO}\left(\mathrm{C}_{5} \mathrm{H}_{9}\right)_{2}\right]^{+}, 100\right\}, 267\left\{\left[\mathrm{M}-\mathrm{PO}\left(\mathrm{C}_{6} \mathrm{H}_{5}\right)_{2}\right]^{+}, \quad 15\right\}, 253$ $\left\{\left[\mathrm{M}-\mathrm{CH}_{2} \mathrm{PO}\left(\mathrm{C}_{6} \mathrm{H}_{5}\right)_{2}\right]^{+}, 16\right\}, 215\left\{\left[\mathrm{CH}_{2} \mathrm{PO}\left(\mathrm{C}_{6} \mathrm{H}_{5}\right)_{2}\right]^{+}, 17\right\}, 201\left\{\left[\mathrm{PO}\left(\mathrm{C}_{6} \mathrm{H}_{5}\right)_{2}\right]^{+}, 63\right\}$. Anal. Calcd. for $\mathrm{C}_{28} \mathrm{H}_{38} \mathrm{O}_{2} \mathrm{P}_{2} \cdot 1.5 \mathrm{H}_{2} \mathrm{O}$ : C, 67.86; $\mathrm{H}$, 8.34. Found: C, 67.74; $\mathrm{H}, 8.19$. 
cis-1-(Diphenylphosphino)-3-[(diphenylphosphino)methyl]cyclopentane (13a). To a solution of $6 \mathbf{a}(100 \mathrm{mg}, 0.21 \mathrm{mmol})$ in degassed $\mathrm{CH}_{3} \mathrm{CN}(5 \mathrm{~mL}), \mathrm{Et}_{3} \mathrm{~N}(0.117 \mathrm{~mL}, 0.84 \mathrm{mmol})$ was added. The mixture was stirred at $0^{\circ} \mathrm{C}$ for $5 \mathrm{~min}, \mathrm{HSiCl}_{3}(0.07 \mathrm{~mL}, 0.69 \mathrm{mmol})$ was added and the mixture was heated under reflux for $3 \mathrm{~h}$. The mixture was allowed to cool to room temperature, degassed benzene $(5 \mathrm{~mL})$ and degassed aqueous solution of $\mathrm{NaOH}(30 \%, 2.2 \mathrm{~mL})$ were added and the mixture was stirred at $60^{\circ} \mathrm{C}$ for $30 \mathrm{~min}$. The mixture was allowed to cool to room temperature, the organic layer was separated, washed with degassed $\mathrm{H}_{2} \mathrm{O}(3 \mathrm{~mL})$, degassed saturated aqueous solution of $\mathrm{NaHCO}_{3}(3 \mathrm{~mL})$ and degassed brine $(3 \mathrm{~mL})$. The organic phase was dried $\left(\mathrm{Na}_{2} \mathrm{SO}_{4}\right)$, filtered and concentrated under reduced pressure, to give 13a as a colorless oil (90 mg, 95\% yield), which was kept under argon. ${ }^{1} \mathrm{H}$ NMR 7,37-7,29 (complex signal, 8H, Ar- $\mathrm{H}_{\text {ortho }}$ ) 7.25-7.20 (complex signal, 12H, Ar- $\mathrm{H}_{\text {meta }}$ Ar- $\mathrm{H}_{\text {para }}$ ), 2.55-2.46 (m, 1H, 1-H), 2.10-2.01 (complex signal, $2 \mathrm{H}, \mathrm{CH}_{2} \mathrm{P}$ ), 1.93-1.80 (complex signal, 3H, 2- $\mathrm{H}_{\alpha}, 3-\mathrm{H}, 4-\mathrm{H}_{\beta}$ or 4$\left.\mathrm{H}_{\alpha}\right), 1.78-1.67(\mathrm{~m}, 1 \mathrm{H})$ and $1.61-1.48(\mathrm{~m}, 1 \mathrm{H})\left(5-\mathrm{H}_{\alpha}, 5-\mathrm{H}_{\beta}\right), 1.39-1.31\left(\mathrm{~m}, 1 \mathrm{H}, 4-\mathrm{H}_{\alpha}\right.$ or $\left.4-\mathrm{H}_{\beta}\right)$, 1.22-1.12 (m, 1H, 2- $\left.\mathrm{H}_{\beta}\right)$; ${ }^{13} \mathrm{C}$ NMR 139.0 (C, d, $\left.{ }^{1} J_{\mathrm{CP}}=11.7 \mathrm{~Hz}\right), 138.8\left(\mathrm{C}, \mathrm{d},{ }^{1} J_{\mathrm{CP}}=11.7 \mathrm{~Hz}\right)$ and $138.6\left(2 \mathrm{C}, \mathrm{d},{ }^{1} J_{\mathrm{CP}}=13.2 \mathrm{~Hz}\right.$ ) (Ar-C $\left.\mathrm{C}_{i p s o}\right), 133.2\left(\mathrm{CH}, \mathrm{d},{ }^{2} J_{\mathrm{CP}}=18.2 \mathrm{~Hz}\right), 133.0\left(\mathrm{CH}, \mathrm{d},{ }^{2} J_{\mathrm{CP}}=18.2\right.$ $\mathrm{Hz}), 132.7\left(\mathrm{CH}, \mathrm{d},{ }^{2} J_{\mathrm{CP}}=18.7 \mathrm{~Hz}\right)$ and $132.5\left(\mathrm{CH}, \mathrm{d},{ }^{2} J_{\mathrm{CP}}=18.2 \mathrm{~Hz}\right)\left(\mathrm{Ar}-\mathrm{CH}_{\text {ortho }}\right), 128.4-128.1$ $\left(\mathrm{CH}\right.$, complex signal, Ar- $\mathrm{CH}_{\text {meta }}$, Ar- $\left.\mathrm{CH}_{\text {para }}\right), 39.7\left(\mathrm{CH}_{2}, \mathrm{dd},{ }^{3} J_{\mathrm{CP}}=19.2 \mathrm{~Hz},{ }^{2} J_{\mathrm{CP}}=8.6 \mathrm{~Hz}, \mathrm{C} 2\right)$, $38.4\left(\mathrm{CH}, \mathrm{dd},{ }^{3} J_{\mathrm{CP}}=13.2 \mathrm{~Hz},{ }^{2} J_{\mathrm{CP}}=8.1 \mathrm{~Hz}, \mathrm{C} 3\right), 35.5\left(\mathrm{CH}, \mathrm{d},{ }^{1} J_{\mathrm{CP}}=8.6 \mathrm{~Hz}, \mathrm{C} 1\right), 34.7\left(\mathrm{CH}_{2}, \mathrm{~d}\right.$, $\left.{ }^{1} J_{\mathrm{CP}}=12.7 \mathrm{~Hz}, \mathrm{CH}_{2} \mathrm{P}\right), 34.1\left(\mathrm{CH}_{2}, \mathrm{dd},{ }^{3} J_{\mathrm{CP}}=8.6 \mathrm{~Hz},{ }^{3} J_{\mathrm{CP}}=6.1 \mathrm{~Hz}, \mathrm{C} 4\right), 29.8\left(\mathrm{CH}_{2}, \mathrm{~d},{ }^{2} J_{\mathrm{CP}}=19.8 \mathrm{~Hz}\right.$, C5); ${ }^{31} \mathrm{P}$ NMR -4.2 [P( $\left.\left.\mathrm{C}_{6} \mathrm{H}_{5}\right)_{2}\right],-20.6\left[\mathrm{CH}_{2} \mathrm{P}\left(\mathrm{C}_{6} \mathrm{H}_{5}\right)_{2}\right]$.

cis-1-(Dicyclopentylphosphino)-3-[(diphenylphosphino)methyl]cyclopentane (13b). From 6b (930 mg, $1.99 \mathrm{mmol}$ ), degassed $\mathrm{CH}_{3} \mathrm{CN}$ (60 mL), Et ${ }_{3} \mathrm{~N}$ (2.5 mL, $\left.17.9 \mathrm{mmol}\right)$ and $\mathrm{HSiCl}_{3}(1.62$ $\mathrm{mL}, 16.1 \mathrm{mmol}$ ) and following the procedure described for 13a, pure 13b was obtained as a yellow oil (920 mg, quantitative yield). ${ }^{1} \mathrm{H}$ NMR 7.43-7.37 (complex signal, 4H, Ar- $\mathrm{H}_{\text {ortho}}$ ), 7.32-7.27 (complex signal, 6H, Ar- $\mathrm{H}_{\text {meta }}$, Ar- $\mathrm{H}_{\text {para }}$ ), 2.17-2.04 (m, 2H, $\mathrm{CH}_{2} \mathrm{P}$ ), 2.03-1.96 (m, $1 \mathrm{H}, 2-\mathrm{H}_{\alpha}$ ), 1.95-1.30 (complex signal, 24H, 8 cyclopentyl- $\mathrm{CH}_{2}$, 2 cyclopentyl-CH, 1-H, 3-H, 4$\left.\mathrm{H}_{\alpha}, 4-\mathrm{H}_{\beta}, 5-\mathrm{H}_{\alpha}, 5-\mathrm{H}_{\beta}\right), 1.18-1.09\left(\mathrm{~m}, 1 \mathrm{H}, 2-\mathrm{H}_{\beta}\right) .{ }^{13} \mathrm{C} \mathrm{NMR} 139.1\left(\mathrm{C}, \mathrm{d},{ }^{1} J_{\mathrm{CP}}=12.1 \mathrm{~Hz}\right)$ and 138.9 $\left(\mathrm{C}, \mathrm{d},{ }^{1} J_{\mathrm{CP}}=12.7 \mathrm{~Hz}\right)\left(\mathrm{Ar}-\mathrm{C}_{i p s o}\right), 132.8\left(\mathrm{CH}, \mathrm{d},{ }^{2} J_{\mathrm{CP}}=20.3 \mathrm{~Hz}\right)$ and $132.5\left(\mathrm{CH}, \mathrm{d},{ }^{2} J_{\mathrm{CP}}=20.3 \mathrm{~Hz}\right)$ (Ar- $\mathrm{CH}_{\text {ortho }}$ ), 128.4-128.2 (CH, complex signal, Ar- $\left.\mathrm{CH}_{\text {meta }}, \mathrm{Ar}-\mathrm{CH}_{\text {para }}\right), 40.1\left(\mathrm{CH}_{2}, \mathrm{dd},{ }^{2} J_{\mathrm{CP}}=11.8\right.$ $\left.\mathrm{Hz},{ }^{3} J_{\mathrm{CP}}=7.6 \mathrm{~Hz}, \mathrm{C} 2\right), 38.1\left(\mathrm{CH}, \mathrm{dd},{ }^{2} J_{\mathrm{CP}}=13.0 \mathrm{~Hz},{ }^{3} J_{\mathrm{CP}}=7.4 \mathrm{~Hz}, \mathrm{C} 3\right)$, 35.4-35.0 (CH, complex signal, cyclopentyl CH, C1), $34.9\left(\mathrm{CH}_{2}, \mathrm{~d},{ }^{1} J_{\mathrm{CP}}=12.1 \mathrm{~Hz}, \mathrm{CH}_{2} \mathrm{P}\right), 33.8\left(\mathrm{CH}_{2}\right.$, dd, ${ }^{3} J_{\mathrm{CP}}=9.0 \mathrm{~Hz}$, $\left.{ }^{3} J_{\mathrm{CP}}=6.7 \mathrm{~Hz}, \mathrm{C} 4\right), 31.1-30.7\left(\mathrm{CH}_{2}\right.$, complex signal, cyclopentyl $\mathrm{C} 2$ and $\left.\mathrm{C} 5\right), 29.7\left(\mathrm{CH}_{2}, \mathrm{~d}\right.$, $\left.{ }^{2} J_{\mathrm{CP}}=15.2 \mathrm{~Hz}, \mathrm{C} 5\right), 26.2-25.8\left(\mathrm{CH}_{2}\right.$, complex signal, cyclopentyl $\mathrm{C} 3$ and $\left.\mathrm{C} 4\right) ;{ }^{31} \mathrm{P}$ NMR 4.1 $\left[\mathrm{PO}\left(\mathrm{C}_{5} \mathrm{H}_{9}\right)_{2}\right],-20.5\left[\mathrm{CH}_{2} \mathrm{PO}\left(\mathrm{C}_{6} \mathrm{H}_{5}\right)_{2}\right]$.

\section{Acknowledgements}

Financial support from the Dirección General de Investigación of Ministerio de Ciencia y Tecnología (project QUI1999-0512) and Comissionat per a Universitats i Recerca of the 
Generalitat de Catalunya (GC) (project 2001-SGR-00085) and a fellowship from the GC (to G. C.) are gratefully acknowledged. We thank the Serveis Científico-Tècnics of the University of Barcelona for NMR and MS facilities and Ms. P. Domènech from the IIQAB (CSIC, Barcelona, Spain) for carrying out the elemental analyses.

\section{References}

1. Camps, P.; Colet, G.; Font-Bardia, M.; Muñoz-Torrero, V.; Solans, X.; Vázquez, S. Tetrahedron 2002, 58, 3473.

2. Camps, P.; Colet, G.; Font-Bardia, M.; Muñoz-Torrero, V.; Solans, X.; Vázquez, S. Tetrahedron: Asymmetry 2002, 13, 759.

3. (a) Conant, J. B.; Braverman, J. B. S.; Hussey, R. E. J. Am. Chem. Soc. 1923, 45, 165. (b) Miller, J. A.; Stewart, D. Tetrahedron Lett. 1977, 1065. (c) Bell, A.; Davidson, A. H.; Earnshaw, C.; Norrish, H. K.; Torr, R. S.; Trowbridge, D. B.; Warren, S. J. Chem. Soc., Perkin Trans. 1 1983, 2879. (d) Mikolajczyk, M.; Zatorski, A. J. Org. Chem. 1991, 56, 1217.

4. Haynes, R. K.; Lam, W. W.-L.; Yeung, L.-L. Tetrahedron Lett. 1996, 37, 4729.

5. (a) Horner, L.; Klink, W. Tetrahedron Lett. 1964, 2467. (b) O'Brien, P.; Warren, S. Tetrahedron Lett. 1995, 36, 2681. (c) Clayden, J.; Warren, S. Angew. Chem. Int. Ed. Engl. 1996, 35, 241. (d) Guéguen, C.; O'Brien, P.; Warren, S.; Wyatt, P. J. Organomet. Chem. 1997, 529, 279. (e) Guéguen, C.; O'Brien, P.; Powell, H. R.; Raithby, P. R.; Warren, S. J. Chem. Soc., Perkin Trans. 1 1998, 3405. (f) Nelson, A.; Warren, S. J. Chem. Soc., Perkin Trans. 1 1999, 1963. 